\title{
Comparison of analytical and numerical methods of obtaining coplanar capacitance of microelectrodes for particulate matter detection.
}

\author{
Pelumi W. Oluwasanya, Member, IEEE, Girish Rughoobur, Member, IEEE, \\ and Luigi G. Occhipinti, Senior Member, IEEE
}

\begin{abstract}
This work reports a comparative analysis between the analytical and the finite element modelling, for the calculation of the capacitance of microelectrode-based capacitors in both parallel and coplanar architecture. It shows that the inverse-cosine and the Schwartz-Christoffel methods underestimate the capacitance value of the coplanar plate capacitor. It also shows that the parallel architecture closed form solution and the finite element modelling results agree well in all cases. The work finally compares the effects of design parameter change of the microelectrodes on the capacitance obtained from each technique. It shows that finite element modelling provides the best estimate of the capacitance of a coplanar plate capacitor.
\end{abstract}

Index Terms-Capacitive PM2.5 sensor; finite element modelling, conformal mapping, inverse-cosine transformation, microelectrodes.

\section{INTRODUCTION}

$\mathrm{C}$ APACITIVE detection has been investigated for real-time monitoring of several parameters such as humidity [1], pressure [2], water level [3] in sensor devices, to mention a few. Recently, the need for miniaturized particulate matter (PM) sensor has seen the development of capacitive PM sensor. Capacitive PM detection technique relies on the fact that an airborne particle close enough to the surface of a charged pair of electrode plates (as shown in Fig. 1) will increase the capacitance of the plates. This increase is a factor dependent on the ratio of volume of the particle to the entire volume of the active space above or between the charged electrodes. The particle displaces air its own volume (for coplanar capacitors and parallel plate capacitor with an air gap). These capacitance jumps for a PM sensor could be detected either with the setup as a single variable capacitor where changes in current flow is measured and calibrated above a certain initial value or as a differential setup where a second capacitor is kept in a

Submitted 29 February 2020. This work was supported in part by the Innovate UK through the projects GraphClean (71476-481865) and MP-SENS (73047501268), by the EPSRC through the Centre for Doctoral Training in Sensor technologies and applications $(\mathrm{EP} / \mathrm{KO} 3099 \mathrm{X} / 1)$, the Centre for Innovative Manufacturing in Large-Area Electronics (EP/LO15889/1), and the Impact Acceleration Account partnership development project MiniPMS (RG 90413). P.W.O. would like to acknowledge support by the Presidential Special Scholarship for Innovation and Development (PRESSID) managed by the National Universities Commission (NUC) and funded by the Petroleum Technology Development Fund (PTDF). controlled environment and connected to the active sensor in such a way that their current flows in opposite directions and only the difference of their current, which represents the contribution of the detected particles is measured. This is the approach presented by Carminati et al. [4]. Generally, there are two architectures for the capacitive PM sensor: parallel or coplanar. The parallel arrangement is very easy to set up and to analyze due to the fact the electric field caused by the charged plates is uniform when sufficiently far from the end points. Though the analysis of the field and the capacitance is very straightforward, this architecture can be difficult to realize and optimize, for instance in PM detection, the inter-plate gap must be at least bigger than the size of the largest particle size the inlet allows. When particles agglomerate, clogging could occur.

The capacitance, $C$, of a parallel plate capacitor is given by closed form solution:

$$
C=\frac{\varepsilon A}{d}
$$

Where $\varepsilon$ is the dielectric constant of the space between the two plates. $A$ is the area of the plates and $d$ is the inter-plate gap.

The potential difference $V$, between the plates is given as:

$$
V=\frac{\sigma}{\varepsilon} \times d
$$

Where $\frac{\sigma}{\varepsilon}$ is the electric field, $E$, neglecting edge effects and $\sigma$ is the charge density.

For PM application, a linear contribution of the volume elements of the inter-plate gap may be assumed. In this case, the change in the dielectric constant leading to a change in capacitance is calculated as follows:

For particles of type $i$ with dielectric constant $\varepsilon=\varepsilon_{0} \varepsilon_{r}$ that displaces air its own volume results in a total dielectric constant

Pelumi W. Oluwasanya is a $\mathrm{PhD}$ candidate in Electrical Engineering Division, University of Cambridge Department of Engineering, $9 \mathrm{JJ}$ Thomson Avenue, Cambridge CB3 0FA.

Girish Rughoobur is a Postdoctoral researcher at Massachusetts Institute of Technology, Department of Electrical Engineering and Computer Science, Cambridge, United States.

Luigi G. Occhipinti is Director of Research in Graphene and Related Technologies at Department of Engineering, University of Cambridge, $9 \mathrm{JJ}$ Thomson Avenue, Cambridge CB3 0FA. Phone: +44 7548 323462, Email: luigi.occhipinti@eng.cam.ac.uk (corresponding author) 
of the space between the electrode plates given by [5]:

$$
\varepsilon_{T}=\left(m \varepsilon_{r}+(1-m)\right) \frac{V_{i}}{V} \varepsilon_{0}
$$

(a)

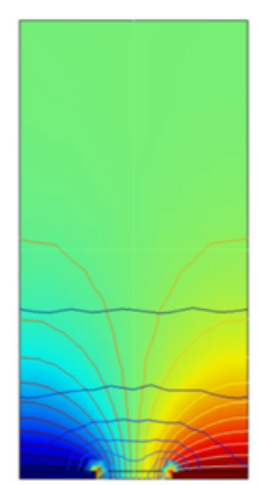

(b)

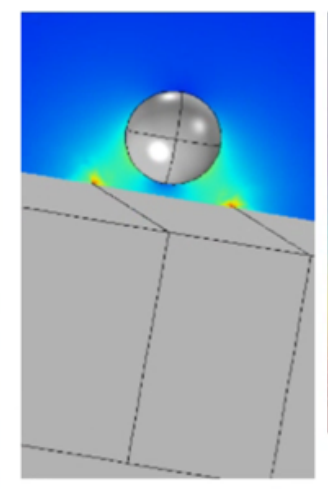

(c)

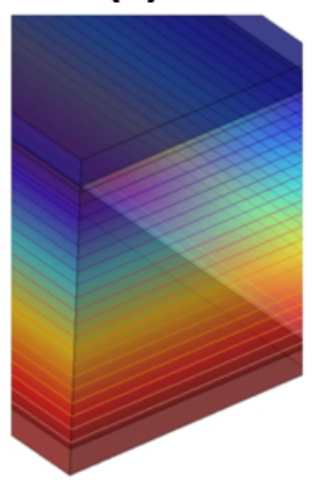

Fig 1. - Capacitive sensor electrodes shown with their field lines for coplanar architecture in (a). 2D and (b). 3D with particle and (c). parallel architecture.

Where $m$ is the number of particles of type $i . V_{i}$ is the volume of a single particle. $V$ is the total volume of the inter electrode gap.

It holds true for both parallel and coplanar electrode plates architectures. However, the highlighted disadvantages of parallel architecture have resulted in the use of the coplanar architecture for PM detection. In this arrangement, two electrode plates are placed horizontally beside each other. The architecture is such that the area of the plates facing the gap is smaller than that of the plate facing the horizontal surface. However, the electric field is not uniform, in fact this is the region of the edge effects which is characterized by field lines clustering at the corners. Thus, intuitively, the fields are stronger towards the edges and corners than in any other part of the surface facing the inter electrode gap. For the sake of sensitivity, the aim would be to get the particles as close to the edges as possible or make the electrodes as thin as possible in the horizontal (width) direction and as much as possible, in number. The second goal can be achieved by using interdigitated electrodes [6] while the first goal, could be achieved using a controlled flow [7].

Calculation of the electric fields, and thus capacitance in the coplanar case is however, not as straightforward as in the parallel case because of the non-uniformity of the electric field distribution. Nonetheless, since the field only varies in twodimensions, the calculation may be simplified. Common approaches for estimating this field have been done using conformal mapping with either Schwarz-Christoffel (SC) transformation [8], [9] or inverse-cosine transformation [10], numerical methods [11] using finite element analysis modelling [12]. These solutions are made simpler because the potential at any point within the boundary and the flux at the same point are perpendicular to each other. Thus, conformal approaches, in which the angle between intersecting lines is preserved, suffice because difficult geometries can be converted into equivalent, more straight-forward ones like the parallel plate architecture for analysis purposes [13].
While conformal mapping approaches are suitable for measurements with high enough signal level such as contacts [15], for high sensitivity measurements such as in portable or Micro-Electromechanical Systems (MEMS)-based capacitive PM sensors [5], [14] where signal levels can be as low as few tens of zeptofarads to a few attofarads, a difference in estimation of the order of a few femtofarads is too large, therefore, conformal mapping approaches may not be suitable. Further, a comparison of the results of each approach is necessary to inform of their limitations in application. Finite element modelling of a capacitive PM sensor has already been described elsewhere [5]. This work evaluates the base capacitance of microelectrodes used in miniaturized sensors such as the PM sensor and shows that while the conformal methods provide a good approximation to the solution, they underestimate the capacitance value and that finite element modelling provides a better estimate as it agrees with the closed form solution for the parallel case.

\section{CONFORMAL MAPPING APPROACHES}

In the inverse cosine case, two semi-infinite coplanar plates (which is a limiting case of cylindrical surfaces) are used to obtain a solution which can then be approximated for the finite plates and proceeds as follows based on findings from Chen et al. [16]. The flux and the potential lines are shown in Fig. 1(a). The transformation [17] is:

$$
W=u+j v=V\left[1-\frac{2}{\pi} \arccos \left(\frac{Z}{d}\right)\right]
$$

Where $u$ and $v$ are defined in volts and coulombs/metre, and $W$ is a complex function of $V$.

When rewritten in terms of $u$ and $v$, differentiated at $y=0$ and substituted:

$$
\frac{\partial u}{\partial y_{y=0}}=\frac{-2 V}{d \pi} 1 / \sinh \left[\frac{\pi}{2 V} v\right]_{y=0}
$$

Which simplifies to: 


$$
Q=\frac{4 \varepsilon_{0} \varepsilon_{r} l V}{\pi} \log \left[\left(1+\frac{w}{d}\right)+\sqrt{\left(1+\frac{w}{d}\right)^{2}-1}\right]
$$

(a)

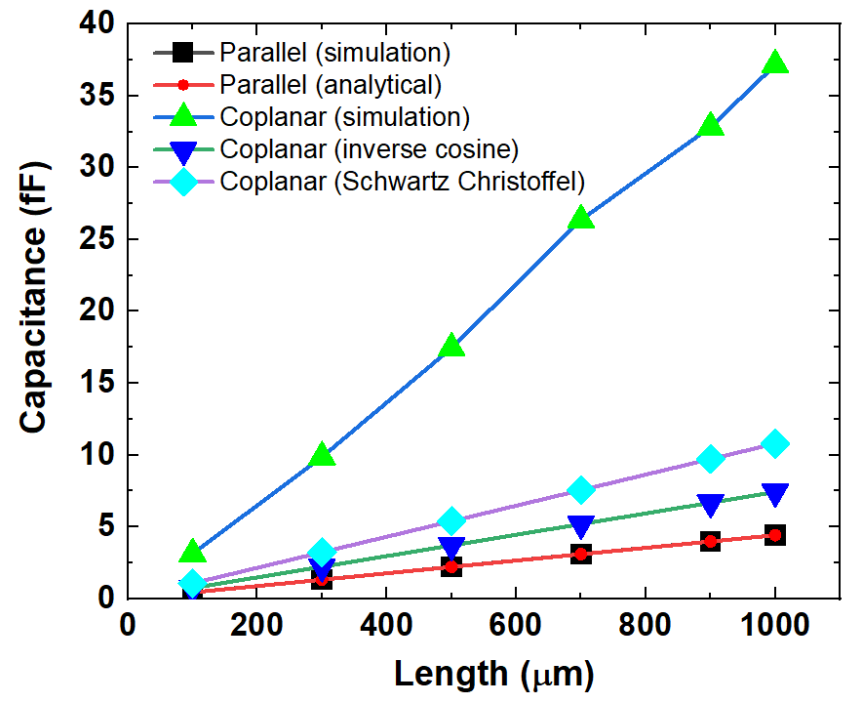

(c)

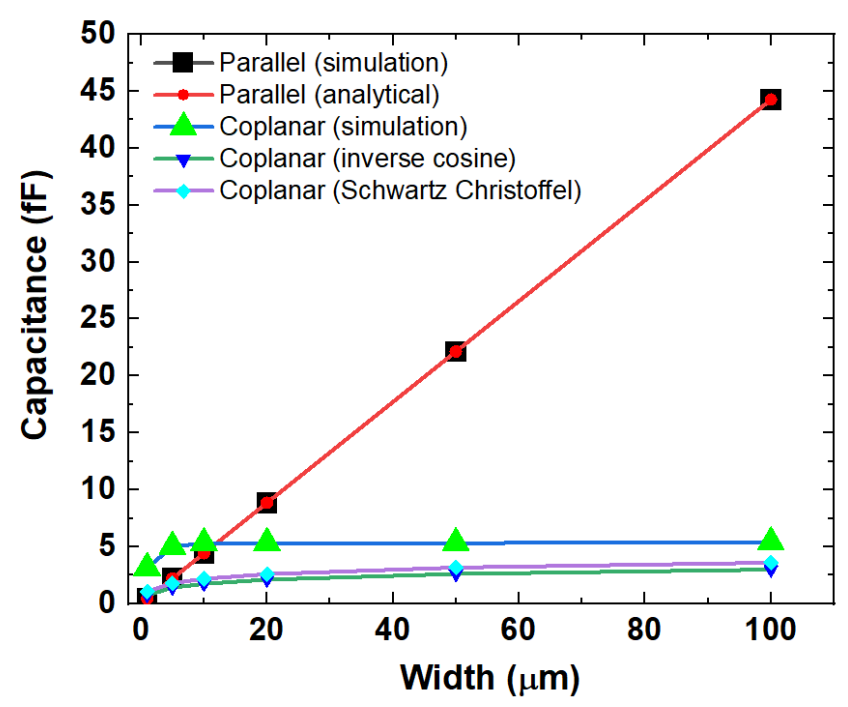

(b)

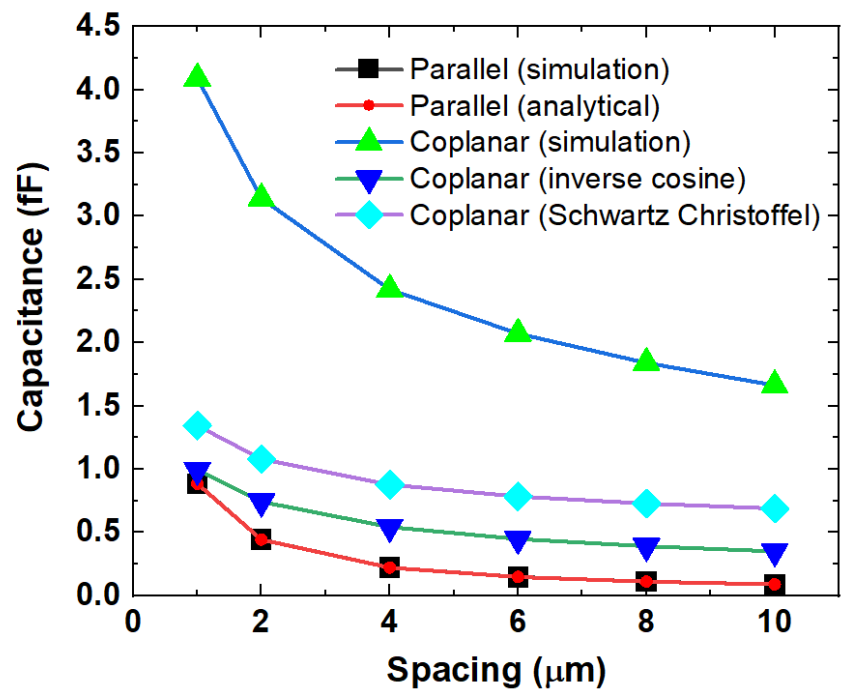

(d)

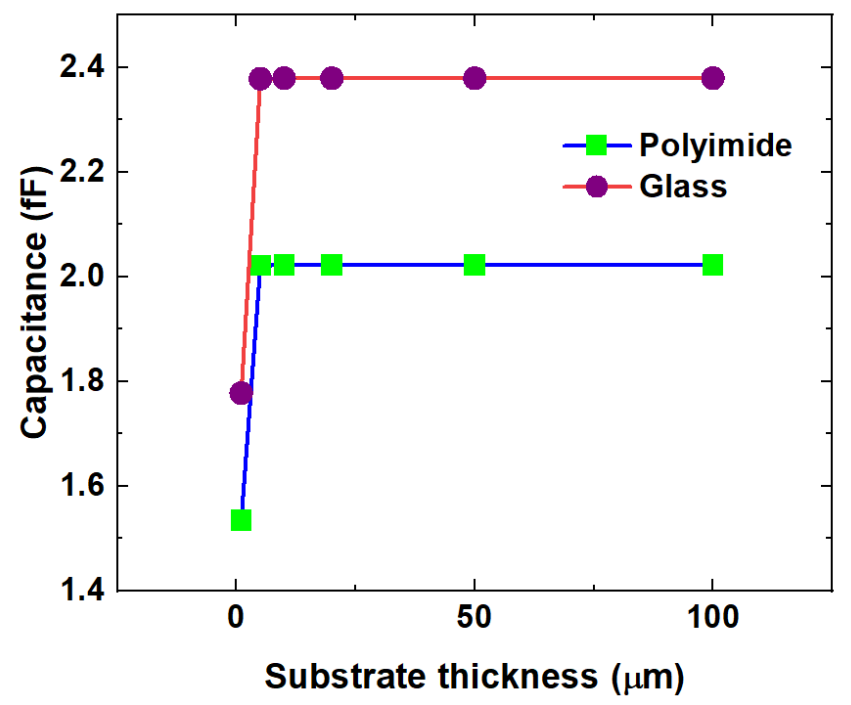

Fig 2. - A comparison of the different approaches for capacitance calculation such as the analytical method, Finite Element modelling method, Schwartz-Christoffel mapping method, and Inverse-Cosine method (a) for varying length of the electrode plates with width $=1 \mu \mathrm{m}$, and spacing $=2 \mu \mathrm{m}$. (b) for varying electrode spacing with length $=100 \mu \mathrm{m}$, and width $=1 \mu \mathrm{m}$ (c) for varying width of the electrode plates with length $=100 \mu \mathrm{m}$, and spacing $=2 \mu \mathrm{m}$. (d) The effect of substrate thickness variation on the capacitance of coplanar capacitors.

For finite length plates, the capacitance is then obtained as:

$$
C=\frac{Q}{2 V}=\frac{2 \varepsilon_{0} \varepsilon_{r} l}{\pi} \log \left[\left(1+\frac{w}{d}\right)+\sqrt{\left(1+\frac{w}{d}\right)^{2}-1}\right]
$$

For the Schwarz-Christoffel transformation approach, the SC transformation maps an arbitrary polygon in the z-plane into a series of segments along the real axis in the $z^{\prime}$-plane through the following equations:

$$
\frac{d W}{d V}=S(V-a)^{\left(\frac{\alpha}{\pi}-1\right)}(V-b)^{\left(\frac{\beta}{\pi}-1\right)}(V-c)^{\left(\frac{\gamma}{\pi}-1\right)}
$$

Where $a, b, c$ are sides of the polygon.

For a rectangle, this becomes:

$\frac{d W}{d V}=S(t-a)^{-\frac{1}{2}}(t-b)^{-\frac{1}{2}}(t-c)^{-\frac{1}{2}}(t-d)^{-\frac{1}{2}}$

Where $a, b, c, d$ are sides of the rectangle.

The equation can be integrated to obtain $W$ as:

$$
W=S \int_{t_{0}}^{t}\left(t^{\prime}-a\right)^{-\frac{1}{2}}\left(t^{\prime}-b\right)^{-\frac{1}{2}}\left(t^{\prime}-c\right)^{-\frac{1}{2}}\left(t^{\prime}-d\right)^{-\frac{1}{2}}
$$

But the similarity between this and the elliptical integral of the 
first kind below can immediately be seen.

\section{Particle trajectories}

(a)

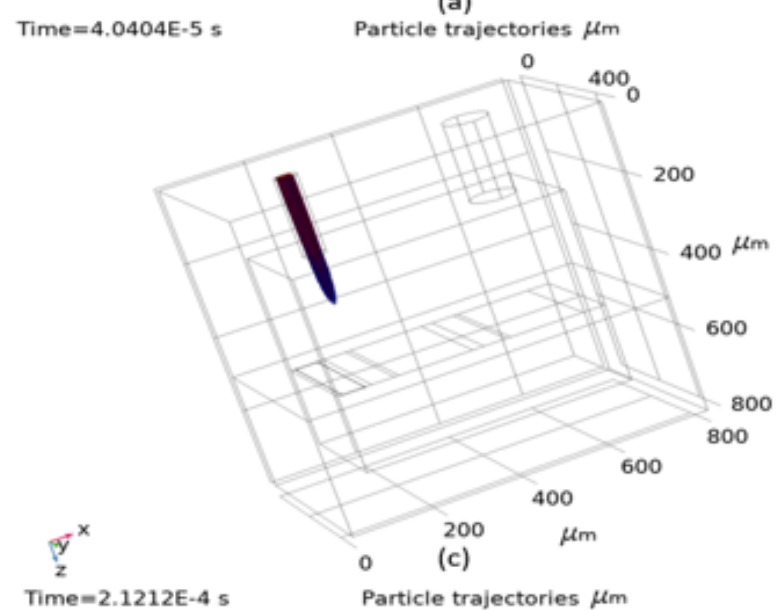

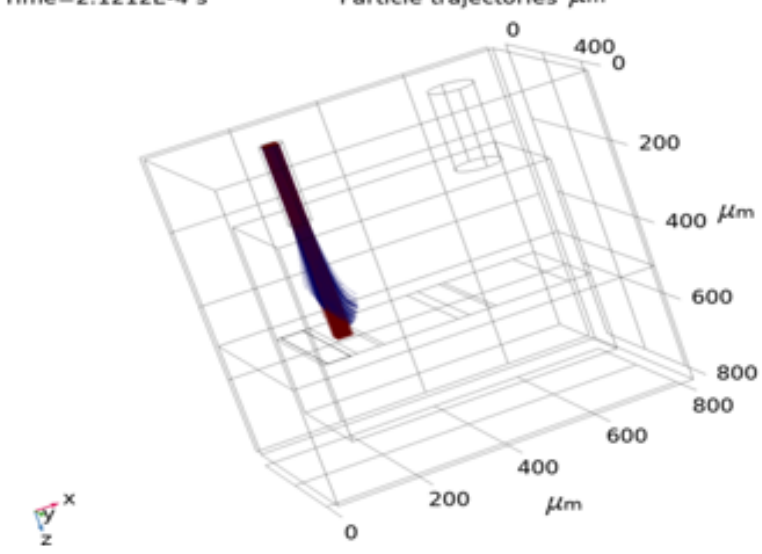

(b)

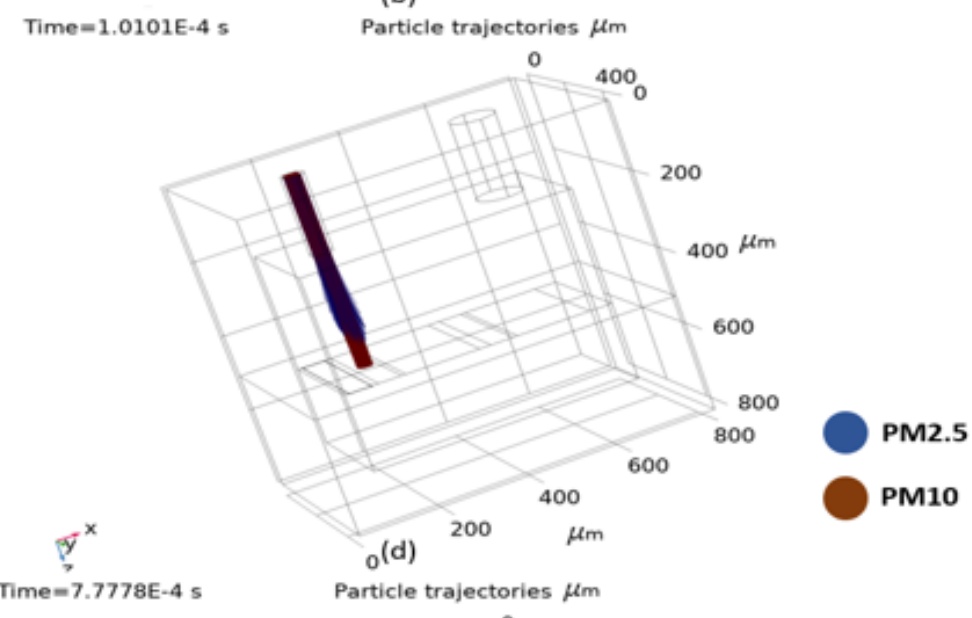

Time $=7.7778 \mathrm{E}-4 \mathrm{~s}$

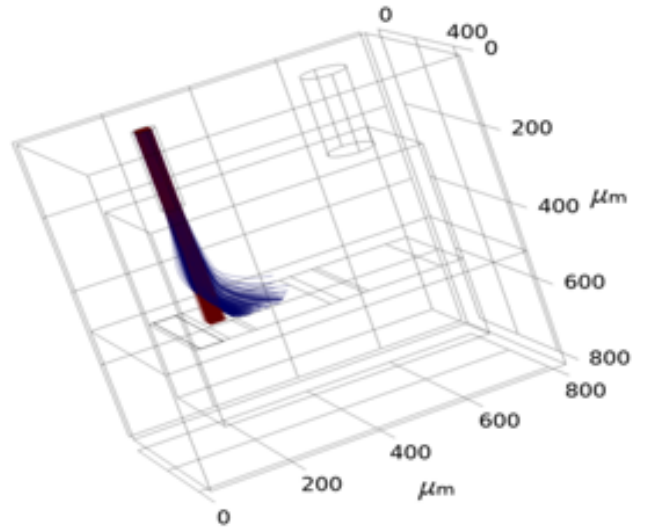

$\mathbb{F}^{x}$

Fig 3. (a) - (d) Simulation results (snapshots of time) showing trajectories of PM2.5 and PM10 particles as they fall vertically on to the surface of the microelectrodes from a single stream they are separated into two streams by thermophoresis and deposited on separate electrodes.

$$
w(t, k)=\int_{0}^{t} d t\left[\left(1-t^{2}\right)\left(1-t^{2} k^{2}\right)^{-\frac{1}{2}}\right.
$$

The goal then is to rewrite the SC mapping as the complete elliptic integral of the first kind to allow for analytical solution to the capacitance calculation. Thus giving:

$$
C=2 \varepsilon_{0} \varepsilon_{r} K(k) / K^{\prime}(k)
$$

And $k$ is then:

$$
k=\frac{w}{w+s}
$$

Which can then be solved by numerical methods as in Sychev et al. [11]. Capacitance $C$, thus calculated is proportional to the area and inter-electrode spacing $A$ calculated as in the parallel plate case as in equation 1 Finally, for the finite element method (FEM),

$$
\nabla \cdot D=\rho_{v}
$$

$$
E=-\nabla V
$$

Where $D$ is the electric displacement and $\rho_{v}$ is the charge density.

All other terms retain their usual meanings.

Following this, each approach was then used to evaluate the capacitance of the microelectrodes with specified dimensions ranging from width, $w=1 \mu \mathrm{m}$ to $100 \mu \mathrm{m}$, inter-electrode spacing, $s=1 \mu \mathrm{m}$ to $10 \mu \mathrm{m}$ and length, $l=100 \mu \mathrm{m}$ to $1000 \mu \mathrm{m}$. A comparison of the results of all the highlighted techniques are then presented in the next section.

\section{COMPARISON OF THE DIFFERENT APPROACHES}

The different approaches for the calculation of capacitance in both parallel and coplanar architectures such as the closed form (analytical) solution of equation 1, finite element modelling for parallel and coplanar architectures, inverse-cosine (IC) and 
Schwartz-Christoffel (SC) mapping options evaluated using the specified equations are shown in the Fig. 2(a) - 2(d). The first one and the last two were done in MATLAB while the FEM was done with COMSOL Multiphysics ${ }^{\circledR}$ tool using the Electrostatics module. In all cases and instances, the analytical and the FEM results for the parallel case were the same as shown in the following figures. However, for the coplanar case, it is shown that both the SC and the IC methods underestimate the capacitance. While the SC results were generally closer to the FEM results for all of the simulations, it can still be seen why these methods are only an approximation. It is however correct for the coplanar capacitances to be higher than the parallel capacitances due to the convergence of the field lines at the edges. Further, Fig. 2(a) shows that as the length of the electrode plates is increased gradually from $100 \mu \mathrm{m}$ to $1000 \mu \mathrm{m}$, the calculated capacitance increases in all cases, the steepest being the coplanar FEM method. The relationship between length and capacitance is linear. The implication of this is that the design constraints will be the main limitations to the sensor design - how big does the capacitor need to be to make for a detectable signal.

The effect of electrode spacing was investigated. The spacing was gradually increased from $1 \mu \mathrm{m}$ to $10 \mu \mathrm{m}$ using a length of $100 \mu \mathrm{m}$ and width of $1 \mu \mathrm{m}$. As shown in Fig. 2(b) the capacitance reduces in all cases and the overall trendline is similar. Thus, the goal in any simulation of fabrication would be to increase the electrode length as long the design permits, increase the electrode width up to the beneficial limit, and also to reduce the spacing as much as possible.

The effect of the increase in width of the electrodes was then investigated. This is shown in Fig. 2(c). The width of the electrodes was gradually increased from $1 \mu \mathrm{m}$ to $100 \mu \mathrm{m}$, while the length was $100 \mu \mathrm{m}$ and the spacing between the electrodes was $2 \mu \mathrm{m}$. For the parallel plates case, as the width of the electrode is increased, the capacitance increased linearly as the equation suggests and the FEM simulation proves. However, for the coplanar case, increase in the electrode width is only beneficial up to around $20 \mu \mathrm{m}$ when further increase in the electrode width does not yield much benefits. This information is very important especially in the design - from the foregoing results on the effects of the length of the electrodes. Therefore, for coplanar electrodes, due to its architecture, and the limitation of its field penetration depth, the capacitance does not increase further beyond the limit. All three coplanar capacitance evaluation techniques utilized agree on this as shown in Fig. 2(c).

Finally, the effect of increasing substrate thickness on the obtained capacitance was investigated for two different substrate materials - glass and polyimide. Glass, with $\varepsilon_{r}=4.2$, as against polyimide's $\varepsilon_{r}=3.4$, had a higher capacitance in all the instances simulated for. The effect of increasing the substrate thickness is negligible after thicknesses higher than $5 \mu \mathrm{m}$ as shown in Fig. 2(d). Thus, instead of increasing the thickness of the substrate continuously, it might be more beneficial to use a different material, such as $\mathrm{Si}_{3} \mathrm{~N}_{4}$ with $\varepsilon_{r}=7.5$.

\section{APPLiCATION TO PARTICULATE MATTER DETECTION}

We applied the coplanar (simulation) method to particulate matter detection to obtain the capacitance jump observed when a particle of $\mathrm{PM}_{2.5}$ falls on the microelectrode surface. The

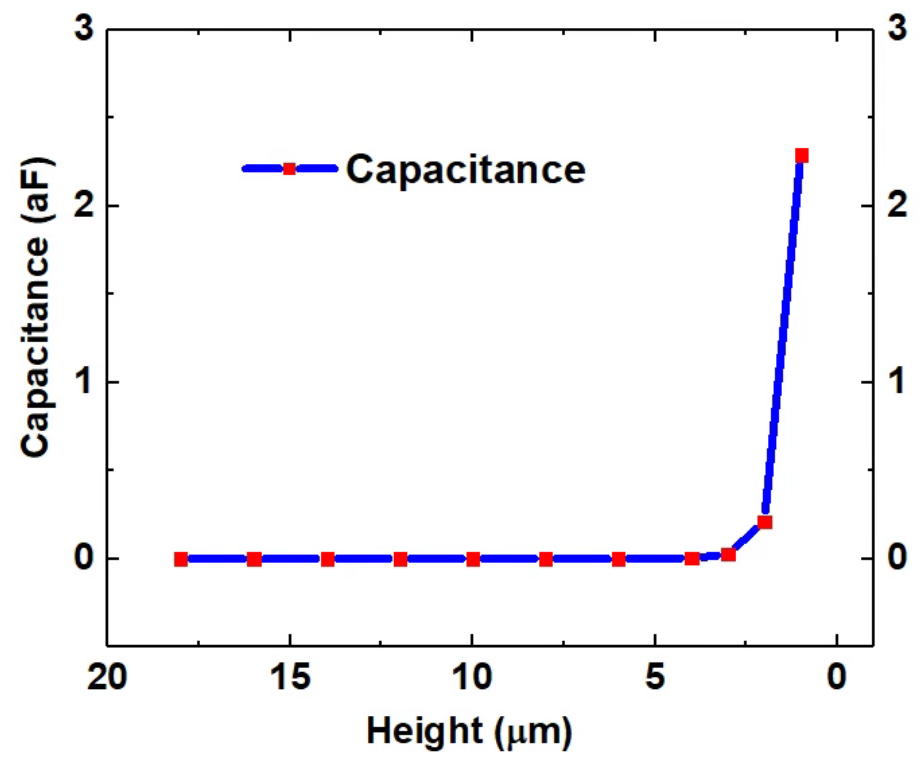

Fig 4. - Capacitive jumps detected for single particle falling vertically on the microelectrodes.

model was developed using the methods specified in our recent work [14]. The particle trajectories obtained are shown in Fig 3. The time steps show the progression of the particles in a MEMS-based device, discriminated by thermophoresis and deposited on different electrodes. Single silica-glass particle of $\varepsilon_{r}=3.75$ and diameter $1.5 \mu \mathrm{m}$ was used in this simulation. The capacitance jumps obtained is shown in Fig 4. From this, it can be deduced that the active volume changes with the dimensions of the electrodes and, in this case, the particle cannot be detected above $4 \mu \mathrm{m}$ compared to $18 \mu \mathrm{m}$ in [5],[14]. The highest capacitance obtained was $2.3 \mathrm{aF}$, which reduces rapidly with height above the microelectrodes.

\section{CONCLUSION}

This work has compared the results of capacitive detection by evaluating the different analytical and FEM options in both the parallel and the coplanar electrode architecture options. The results presented show that the for the parallel case, the simulation and the analytical results are exactly the same; however, for the coplanar case, inverse cosine and SchwartzChristoffel mapping options underestimate the capacitance and therefore, the better approach would be to use FEM. Finally, the results were then used to simulate a MEMS-based system for particulate matter detection with sensitivity to single particles.

\section{ACKNOWLEDGMENT}

L.G.O. and P.W.O. would like to thank the Innovate UK for their support through the projects GraphClean (71476-481865) and MP-SENS (73047-501268), the EPSRC for their support through the Centre for Doctoral Training in Sensor technologies 
and applications (EP/KO3099X/1), the Centre for Innovative Manufacturing in Large-Area Electronics (EP/LO15889/1), and the Impact Acceleration Account partnership development project MiniPMS (RG 90413). P.W.O. would like to acknowledge support by the Presidential Special Scholarship for Innovation and Development (PRESSID) managed by the National Universities Commission (NUC) and funded by the Petroleum Technology Development Fund (PTDF).

\section{REFERENCES}

[1] C. M. K. Gardner, T. J. Dean, and J. D. Cooper, "Soil water content measurement with a high-frequency capacitance sensor," J. Agric. Eng. Res., vol. 71, no. 4, pp. 395-403, 1998.

[2] Rud, "United States Patent (19)."

[3] A. Jaworek, A. Krupa, and M. Trela, "Capacitance sensor for void fraction measurement in water/steam flows," Flow Meas. Instrum., vol. 15, no. 5-6, pp. 317324, Oct. 2004.

[4] M. Carminati, L. Pedalà, E. Bianchi, F. Nason, G. Dubini, L. Cortelezzi, G. Ferrari, and M. Sampietro, "Capacitive detection of micrometric airborne particulate matter for solid-state personal air quality monitors," Sensors Actuators, A Phys., vol. 219, pp. 80-87, 2014.

[5] P. W. Oluwasanya, G. Rughoobur, and L. G. Occhipinti, "Design, Modelling and Simulation of a Capacitive size-discriminating Particulate Matter Sensor for personal air quality monitoring," IEEE Sens. J., pp. 1-1, 2019.

[6] S. S. Gevorgian, T. Martinsson, P. L. J. Linnér, and E. L. Kollberg, "CAD models for multilayered substrate interdigital capacitors," IEEE Trans. Microw. Theory Tech., vol. 44, no. 6, pp. 896-904, 1996.

[7] P. W. Oluwasanya, A. Alzahrani, V. Kumar, Y. A. Samad, and L. G. Occhipinti, "Portable multi-sensor air quality monitoring platform for personal exposure studies," IEEE Instrum. Meas. Mag., vol. 22, no. 5, pp. 36-44, Oct. 2019.

[8] S. Ramo, J. R. Whinnery, and T. Van Duzer, Fields and
[14] P. W. Oluwasanya, A. Alzahrani, V. Kumar, and L. G. Occhipinti, "Modelling and Simulation of a portable, size-discriminating Capacitive Particulate Matter sensor," in FLEPS 2019 - IEEE International Conference on Flexible and Printable Sensors and Systems, Proceedings, 2019.

[15] F. Stellari and A. L. Lacaita, "New formulas of interconnect capacitances based on results of conformal mapping method," IEEE Trans. Electron Devices, vol. 47, no. 1, pp. 222-231, Jan. 2000.

[16] J. Z. Chen, A. A. Darhuber, S. M. Troian, and S. Wagner, "Capacitive sensing of droplets for microfluidic devices based on thermocapillary actuation," R. Soc. Chem. , no. 4, pp. 473-480, 2004.

[17] J. R. Brock, "On the theory of thermal forces acting on aerosol particles," J. Colloid Sci., vol. 17, pp. 768-780, 1962.

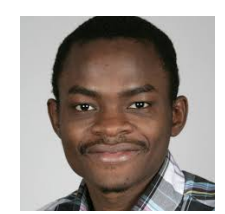

Mr Pelumi W. Oluwasanya is a PhD candidate in Engineering at Cambridge University. He has a BSc (Hons) in Electrical/Electronic Engineering from Olabisi Onabanjo University with First Class, a MSc in Signal processing and communications from The University of Edinburgh and a MRes in Sensor Technologies and Applications from the University of Cambridge. His research focus is on sensors; their design using new materials, fabrication and applications in air quality monitoring, wearables and healthcare.

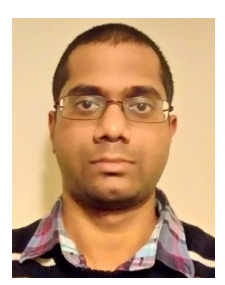

Dr Girish Rughoobur is at the Microsystems Technology Laboratories, Massachusetts Institute of Technology (MIT), USA as a Research Associate. He is currently working on improving the performance of Si field emission arrays in low vacuum for a wide range of applications. He has a MEng in Electrical and Electronic Engineering from the University College London (UCL), UK in 2012. Girish obtained a PhD in Engineering in 2017 from the University of Cambridge, UK for his work on the fabrication and characterization of in-liquid thin film bulk acoustic resonators for sensors/biosensors. Girish is also a member of the Institution of Engineering and Technology and the Institution of Electrical and Electronics Engineers. 


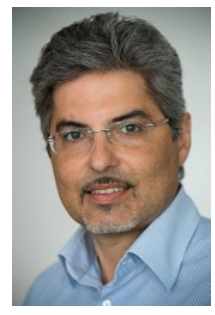

Dr Luigi G. Occhipinti is the Director of Research in Graphene and Related Technologies and Principal Investigator at the University of Cambridge, Department of Engineering. He has developed science and innovation for more than 20 years in emerging technology areas for the post-CMOS roadmap, smart systems heterogeneous integration, polymer and printed electronics, advanced bio-systems and molecular diagnostics, advanced signal processing and nonlinear computation, advanced mechanical, optical and chemical sensor technologies, with focus on miniaturization and advanced packaging technologies for smart devices and products. He is the Deputy Director and Chief Operating Officer of the Cambridge Graphene Centre and the MRes Director of the EPSRC Centre in Graphene Technology. 